\title{
USING AGGREGATED DISCRETE EVENT SIMULATION MODELS AND MULTI-OBJECTIVE OPTIMIZATION TO IMPROVE REAL-WORLD FACTORIES
}

\author{
Simon Lidberg \\ Leif Pehrsson \\ Amos H.C. Ng \\ School of Engineering Science \\ University of Skövde \\ Skövde, 541 28, SWEDEN
}

\begin{abstract}
Improving production line performance and identifying bottlenecks using simulation-based optimization has been shown to be an effective approach. Nevertheless, for larger production systems which are consisted of multiple production lines, using simulation-based optimization can be too computationally expensive, due to the complexity of the models. Previous research has shown promising techniques for aggregating production line data into computationally efficient modules, which enables the simulation of higher-level systems, i.e., factories. This paper shows how a real-world factory flow can be optimized by applying the previously mentioned aggregation techniques in combination with multi-objective optimization using an experimental approach. The particular case studied in this paper reveals potential reductions of storage levels by over $30 \%$, lead time reductions by $67 \%$, and batch sizes reduced by more than $50 \%$ while maintaining the delivery precision of the industrial system.
\end{abstract}

\section{INTRODUCTION}

The current state of the automotive industry is one of major change and transformation. Continuously shorter product life-cycles with reduced implementation times, increasing number of variants, and disruptive new technologies shape the industry. These challenges require current and future production systems to be more flexible and re-configurable. At the same time the lead time will need to be reduced while keeping internal and external customers sated (Koren 2010). One way of supporting companies to meet these issues and challenges is higher quality decision-support, which also can be delivered faster than previously has been possible. Currently, the established way of making predictions about future performance of production systems is using discrete-event simulation (DES) models (Schruben 2010).

These models tend to increase in complexity through their life-cycle in order to be able to answer complex questions about the system. Reducing model complexity has been studied in previous work by Pehrsson et al. (2015), and the same technique has been applied to manually experiment with safety levels to reduce storage levels and lead time (Lidberg et al. 2018). Reducing both lead-time and storage sizes while maintaining a high delivery precision reduces the cost of inventory and time to market and is of great importance for many companies (Hopp and Spearman 2011). Utilizing multi-objective optimization has the potential to move key parameters closer to their ideal objective values, but also enables new insights to be gained about the behavior of the industrial system. The process of improving simulation models, and in extension real physical systems, combining DES and multi-objective optimization is commonly referred to as simulation-based multi-objective optimization (SMO).

This paper presents the application of SMO on aggregated simulation modeling in a real world industrial setup with results of lowering the storage levels by $31 \%$, lead time by $67 \%$, and batch sizes by more than 


\section{Lidberg, Persson, and $\mathrm{Ng}$}

$50 \%$, showing the potential of optimizing on the factory level. The paper begins with a theory section for discrete event simulation, aggregation techniques and multi-objective optimization. Using these concepts, an aggregated model will be constructed for an industrial system to be used in an optimization setup. The results of the optimization will be presented and compared to the manual settings from our previous work. Insights from the optimization will be used to improve the industrial system. Finally, the paper is ended with conclusions and suggestions for future work.

\section{AGGREGATED MODELS IN SMO}

Focusing on the optimization level of SMO and describing in short how aggregation techniques can be applied to complex DES models, this section is comprised of two parts. The first part deals with simulation, modeling, and the possibility of reducing the computational time, data collection time and time required for modeling by aggregating data and logic. The second part summarizes how optimization using genetic algorithms can be applied to find solutions to many-objective problems.

\subsection{DES and Aggregated Modeling}

Building models to represent an already existing physical system or planned system is now common practice in many industries (Negahban and Smith 2014; Banks et al. 2009). The models are used to improve or verify system solutions for either future lines or current ones. Testing proposed changes in models before implementing them physically increases the confidence and robustness of the proposed solution and reduces the likelihood of implementing costly mistakes.

Different questions stated to the model requires different levels of model detail and have different requirements for input data. Adding detail often means that the computational time will increase when running the model, but also that the simulation project duration will be extended for gathering data (Chwif et al. 2000; Fowler and Rose 2004).

To enable faster delivery of decision support to the decision maker one approach is reducing the level of details in the models and thereby reducing the computational time. Several methods have been developed to aggregate models by both mathematical and hybrid modeling (Zulkepli et al. 2012; Lefeber and Armbruster 2011; Asmundsson et al. 2006). With aggregation through DES modeling, reducing the requirements of input data, and while maintaining the characteristics of a detailed model, one approach has been developed by Pehrsson et al. (2014) and Pehrsson et al. (2015). Using this method, the only input parameters required for the aggregation of production lines are processing time, availability, mean time to repair, average work in process (WIP), maximum WIP, and minimum lead time.

\subsection{Simulation-Based Optimization for Many Objectives Using Genetic Algorithms}

Manual experimentation or design of experiment (DOE) studies have several limitations. Preparing experiments is time-consuming and an exhaustive iteration through the design parameters will lead to increasingly large experiment sets. In the effort to contain the size of the experiment sets, limits are imposed to the experiment inputs, which also impose limits on the insights gained from the results.

Genetic algorithms can be used to optimize these settings without limiting the inputs (Floreano and Mattiussi 2008). Letting the algorithm, preferably without external bias, explore the search space will lead to better results which are also non-intuitive (Deb 2001). NSGA-II is a multi-objective genetic optimization algorithm which has been applied frequently in SMO due to its applicability, accuracy, convergence and diversification of solutions on the Pareto front (Deb et al. 2002; Dudas et al. 2014; Ng et al. 2011).

The most recent development has produced algorithms such as NSGA-III which has exhibited improved performance for more than three objectives compared to other algorithms (Deb and Jain 2014; Jain and Deb 2014). The algorithm depends on user-supplied or calculated reference points in the objective space. These points are placed on a normalized hyper-plane constructed for all objectives, and for each solution the distance to these points are minimized instead of selection by maximizing the crowding distance to 
neighbors as in NSGA-II. Selection by proximity to the reference points will increase the diversification of the solutions.

\section{MODEL FOR THE INDUSTRIAL SYSTEM AND OPTIMIZATION SETUP}

This section describes the industrial system on a higher level and how the model is constructed which represents the industrial system. The scheduling and ordering systems implemented in the model will be discussed, because they differ from the real industrial case. Lastly, the setup of the optimization will be explained, the different inputs to change, the outputs to measure, and goals to improve.

\subsection{Industrial System and Model Setup}

This paper shares the same type of industrial setup presented in previous work (Lidberg et al. 2018). A number of issues are limiting the performance in the current setup of the system. Deviating capacities, large batch sizes, uneven distribution of shifts, and large amounts of inventory result in long lead times through the plant. The operation's management requested more information, based on simulation and optimization, in order to enable more informed decision-making on how to improve the situation. The industrial systems consist of several machining lines (ML), producing four different component types, each comprised of several variants. These components are assembled in one of two automated assemblies (AA), and then transported into one of four final assemblies (FA). Between each of these steps, and after FA, are final good inventories (FGI). To model this many interconnected systems in detail would be very computationally expensive to run. Each line is therefore modelled using an aggregation technique, referenced in Section 2.1, to obtain decision-support in a short amount of time. The largest portion of the simulation time is currently the scheduling logic that is implemented in the model, which is not connected to the aggregation technique.

Figure 1 shows the setup of the industrial system, but also how the model is constructed. Each ML is named A-D depending on the component they produce and $\{1, \ldots, N\}$ to denote the number of lines for that component. AA and FA lines are only named iteratively with $\{1, \ldots, N\}$ where AA01 delivers to FA01-02 and AA02 delivers to FA03-04. Input and output data from the model has been obfuscated to protect company assets and are in some cases generalized due to the need for brevity. However, all data relations has been preserved.

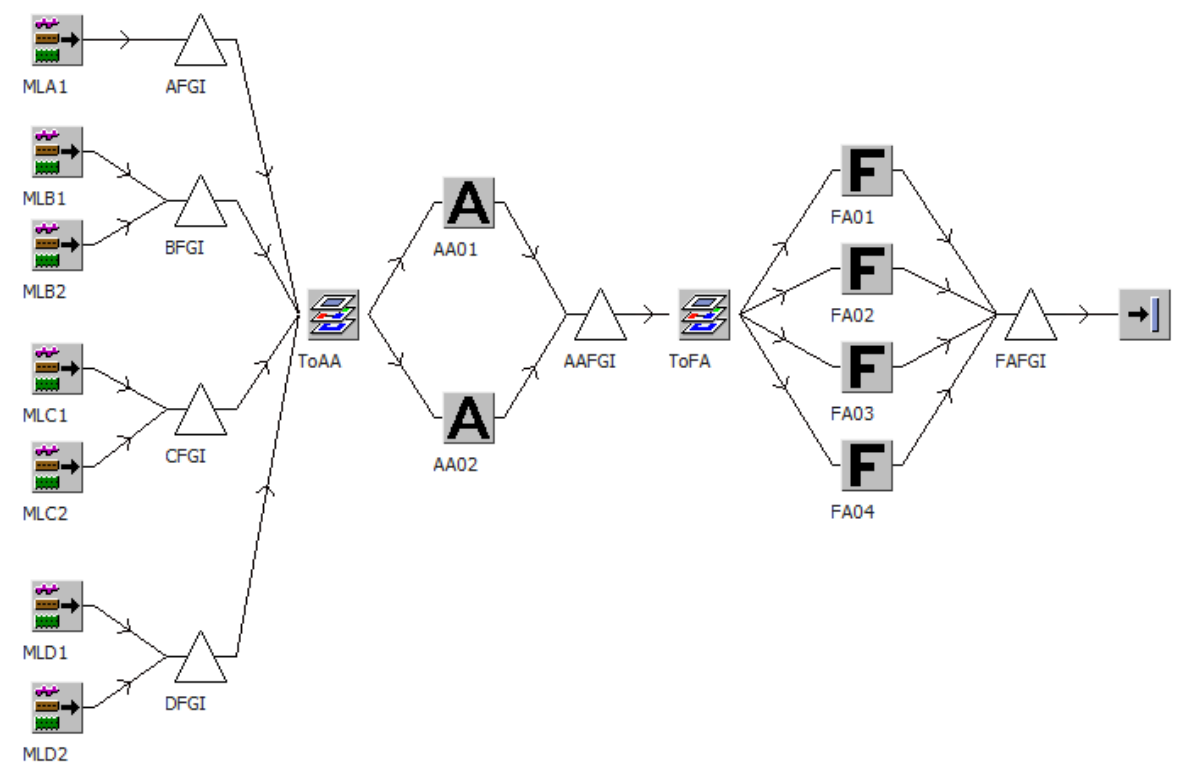

Figure 1: High-level representation of the industrial system and setup of the model. 


\section{Lidberg, Persson, and $\mathrm{Ng}$}

MLC lines are affected by a setup time between the different variants leading to a large batch size and therefore a large storage size. Setup times changing from one variant to another, regardless of the pairing, has been estimated to be 40 minutes. The setup takes place at the LineInput and LineOutput objects of the ML when applied in the aggregation technique. These objects are two of the five objects in the modeling technique and they are separated by a LineWIP object. An implementation is shown in Figure 2 below. Controlling the WIP is handled by a pallet system where the total number of pallets is set by the maximum WIP parameter mentioned earlier. At LineInput the WIP pallets are assembled with incoming products which are passing through the system. When exiting LineOutput, the pallets are passed to WIPControl and the products are sent to the next step in the process, labelled Out.

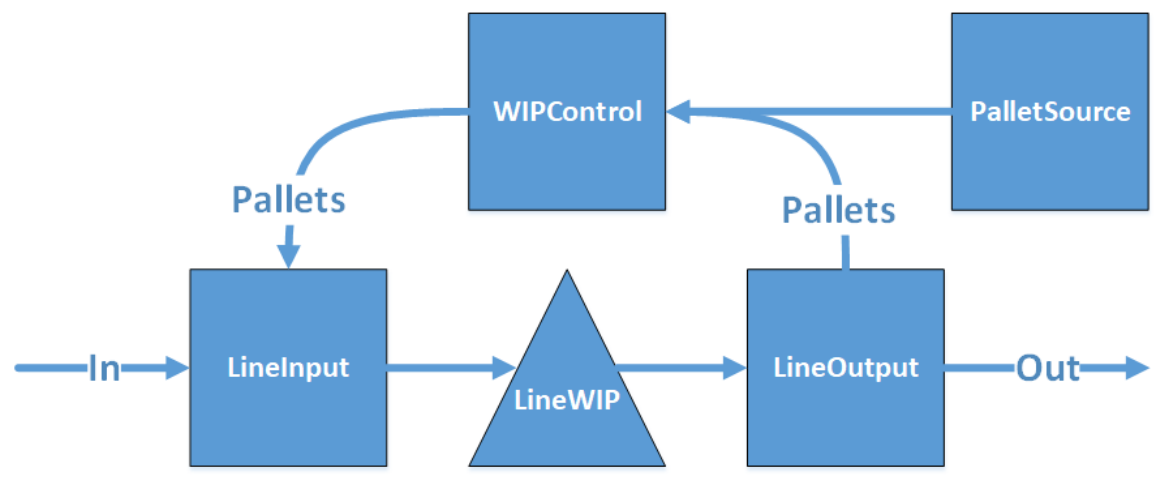

Figure 2: Schematic description of the aggregation technique.

Scheduling principles included in the model consists of a safety stock and order point system from the FGI A-D into AA. This constitutes a pull flow where orders are only started in ML if there is a need from AA. From AA and forward, the products will be assembled using a push system and be routed, depending on the type of product, to the correct FA. Dispatching from FAFGI to the customer follows a fixed schedule of departures and is setup to deliver 13200 products each week running on weekdays only. Order data is extracted from the real-world planning system.

The model is constructed using Siemens Tecnomatix Plant Simulation version 14.0.2. The simulation horizon is 28 days, where 7 days is warm-up time for the model, this will mean that the total target number of products delivered is 39600 . To get statistically sound results, four replications are performed for every evaluation.

\subsection{Optimization Settings}

In order to reduce the total storage size and reduce lead time, lowering the safety level $\alpha$ of each variant $i$, for each storage $S \in\{A, B, C, D, A A, F A\}$, is crucial when considering the type of order system used in the model. The machining storages and components are represented by $m=\{A, B, C, D\}$ where $m \subseteq S$. The minimum, maximum, and step size for these parameters is formulated in (1).

$$
\alpha_{r i}=\left\{\begin{array}{l}
i \in\{1, \ldots, 4\} \wedge r \neq B, \\
i \in\{1, \ldots, 7\} \wedge r=B
\end{array} \quad \text { where } \alpha_{r i} \in\{1, \ldots, 20\}, r \in m \text { and } \Delta \alpha_{r i}=1\right.
$$

Batch sizes $\beta_{C i}$, for component $C$ and each variant $i$, signifies the third input to be changed in the optimization. Due to the time it takes to setup between different variants of component $C$, the production lines need to be run with a larger batch size. This in turns affects the total storage size because of the need to increase the safety stock levels to be able to replenish an article before depletion. $\beta_{C i}$ also controls the minimum amount to start in the line, multiple batches can be queued to reach the production targets.

$$
\beta_{C i} \in\{32, \ldots, 2048\} \text { where } i \in\{1, \ldots, 4\} \text { and } \Delta \beta_{C i}=32 \text {. }
$$




\section{Lidberg, Persson, and $\mathrm{Ng}$}

The last input parameter is an experiment variable $\gamma_{i}$, where $i \in\{1, \ldots, 4\}$, which controls how the shifts and paces are divided among the production lines. By improving the synchronization in the plant, running assembly on additional time with less jobs per hour (JPH), less strain is put on the machining areas leading to a possible reduction in safety levels (Hopp and Spearman 2011). The parameter operates on 4 levels, described in Table 1. Each column, 1-4, for $\gamma_{i}$ represents the number of shifts used and which capacity used for that shift. Shift 1 represents the day shift, Shift 2 represents the evening shift and Shift 3 represents the night shift, each comprising 40 hours per week. On the weekend, Shift 4 operates 24 hours, consisting of day time, and Shift 5 comprises the night time but is omitted here. AA01 moves from using three shifts with an unbalanced pace in $\gamma_{1}$ to a balanced pace over four shifts in $\gamma_{4}$. On the other hand, FA01 is not utilized in $\gamma_{2}$ and $\gamma_{4}$, indicated by the lack of paces. Only changes in AA and FA are accounted for here, machining lines are already performing at maximum capacity, over a number of shifts.

Table 1: Target JPH for each shift per line. Every active shift will have a JPH associated to it.

\begin{tabular}{|c|c|c|c|c|c|c|c|c|c|c|c|c|c|c|c|c|}
\hline \multirow[b]{2}{*}{ Shift } & \multicolumn{3}{|l|}{$\gamma_{1}$} & \multirow[b]{2}{*}{4} & \multicolumn{3}{|l|}{$\gamma_{2}$} & \multirow[b]{2}{*}{4} & \multicolumn{3}{|l|}{$\gamma_{3}$} & & \multicolumn{3}{|l|}{$\gamma_{4}$} & \multirow[b]{2}{*}{4} \\
\hline & 1 & 2 & 3 & & 1 & 2 & 3 & & 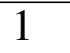 & 2 & 3 & & 1 & 2 & 3 & \\
\hline AA01 & 3 & 37 & 15 & & 30 & 30 & 30 & & & 30 & 30 & & 27 & 27 & 27 & 27 \\
\hline AA02 & 41 & 41 & 34 & & 33 & 33 & 33 & 33 & 33 & 33 & 33 & 33 & 33 & 33 & 33 & 33 \\
\hline FA01 & 20 & 20 & & & & & & & 10 & 10 & 10 & & & & & \\
\hline FA02 & 1 & 17 & 15 & & 30 & 30 & 30 & & 20 & 20 & 20 & & 27 & 27 & 27 & 27 \\
\hline 03 & 21 & 21 & 21 & & 21 & 21 & 21 & 21 & 21 & 21 & 21 & 2 & 21 & 21 & 21 & 21 \\
\hline FA04 & 20 & 20 & 13 & & 12 & 12 & 12 & 12 & 12 & 12 & 12 & 12 & 12 & 12 & 12 & 12 \\
\hline
\end{tabular}

For outputs, average lead time in hours per product $L T_{S}$, average storage level for each storage $W I P_{S}$, number of products delivered NumOut, and total number of hours waiting for components for each AA $W C_{k m}$. Where $k \in\{1,2\}$ represents AA01 and AA02 respectively. The first objective (3) represents the maximum average lead time for the machining components, with the average lead time for AA and FA added. The second objective (4) minimizes the total amount of storage in the model. Lead times and storage sizes are highly influenced by changes to $\alpha_{r i}, \beta_{C i}$, and paces in $\gamma_{i}$. The third and fourth objective, (5) and (6) respectively, operate on one output each. The last objective (6) ensures that the solution delivers the right amount of products to the customer.

$$
\begin{gathered}
\min L T \text { Plant }=\max _{r \in m}\left\{L T_{r}\right\}+L T_{A A}+L T_{F A} \\
\text { minLeanBuffer }=\min \left\{\sum_{j \in S} W I P_{j}\right\} \\
\text { minWaitingParts }=\min \left\{\sum_{k=1}^{2} \sum_{r \in m} W C_{k r}\right\} \\
\text { maxOut }=\max \{\text { NumOut }\} .
\end{gathered}
$$

The optimization will be run using NSGA-III, due to the number of objectives used. Table 2 lists the settings for the algorithm and calculating the number of reference points follows the approach from the original paper (Deb and Jain 2014; Das and Dennis 1998). 
Lidberg, Persson, and $\mathrm{Ng}$

Table 2: Settings for the NSGA-III algorithm used in the optimization.

\begin{tabular}{ll}
\hline Parameter name & Setting \\
\hline Population size & 100 \\
Reference points & 84 \\
Mutation type & Polynomial \\
Mutation distribution index & 5 \\
Mutation probability & 0.041667 \\
Crossover type & SBX \\
Crossover distribution index & 20 \\
Crossover probability & 0.9 \\
\hline
\end{tabular}

\section{RESULTS}

The optimization was set to run until 40000 evaluations had been performed and all the results are shown in Figure 3 using a parallel coordinates chart. The first settings are the objectives followed by the inputs $\gamma_{i}$ to $\beta_{C 4}$. Only few insights can be gained from presenting this data unformatted, mainly regarding the maximum and minimum values of the input and objective values. Interesting to note is that due to the setup of the model, every result not reaching 39600 for maxOut could be considered infeasible.

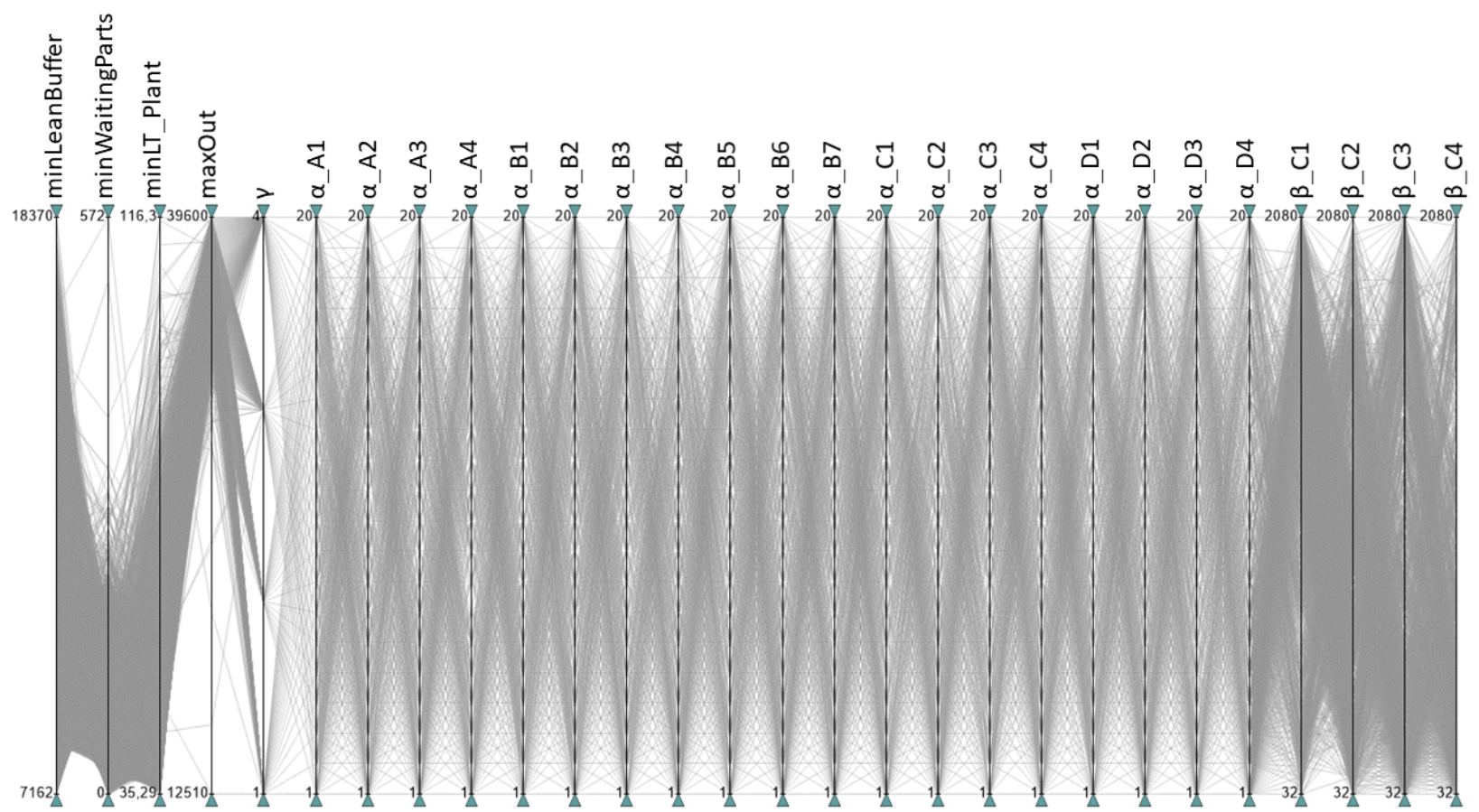

Figure 3: The entire solution set from the optimization in a parallel coordinates chart.

For Figure 4, the results are filtered on maxOut reaching 39 600. A coloring scheme is also applied to highlight the different settings for $\gamma_{i}$, where $\gamma_{2}$ and $\gamma_{3}$ are considered to represent the same effect. Contrasting $\gamma_{1}$ and $\gamma_{4}$, representing the maximum unlevelled flow and the maximum levelled flow respectively, $\gamma_{1}$ has better performance for minLeanBuffer and $\gamma_{4}$ performs better in minLT_Plant. Increasing the number of shifts will reduce the lead time by itself, on the condition that a customer exists, because lead time in the model is based on calendar time. 


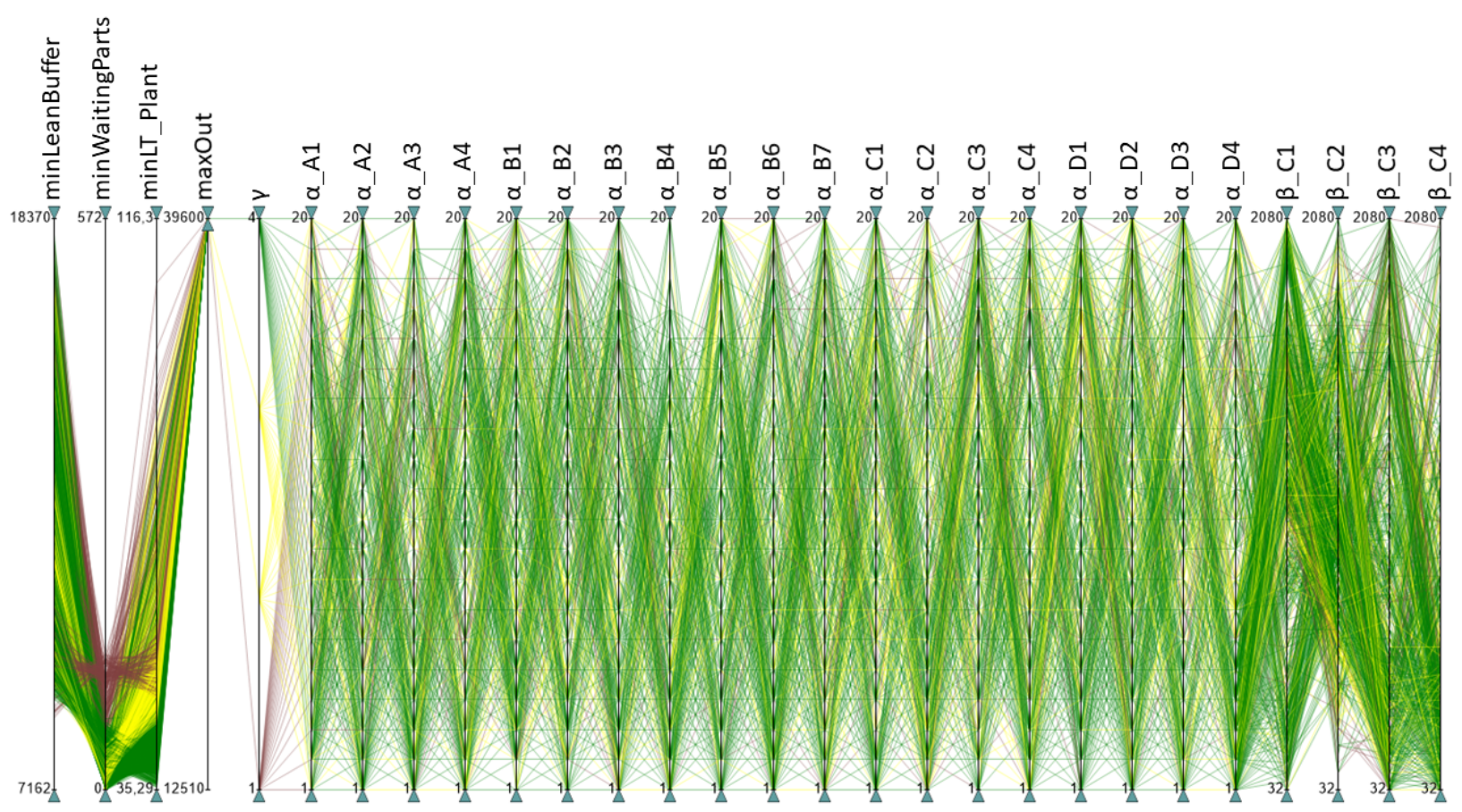

Figure 4: Filtered on delivery target and colored by $\gamma$.

To further analyze the results, a Non-Dominated Sorting (NDS) filter was applied where only the bestsolutions on the Pareto front are selected. This is shown in Figure 5, where the filtering from Figure 4 is kept active. This nearly removes all solutions where $\gamma_{1}$ is used, and should minWaitingParts be considered, $\gamma_{1}$ can be removed entirely. The high settings for $\alpha_{B 5}$ and $\alpha_{C 3}$ are explained since they are low volume variants and increasing the safety level for either results in a minor increase in storage levels, thereby not affecting the objectives sufficiently for them to be minimized.

Finally, by limiting the values for the largest batch sizes, $\beta_{C 1}$ and $\beta_{C 2}$, the objectives minLeanBuffer and minWaitingParts are automatically reduced. The lowest results for minWaitingParts are not possible to reach with this configuration, because setup takes up a larger portion of the production time. Considering only these attributes, the setup time and batch size can be considered to be the bottleneck for further reducing storage size and lead time. In Figure 6, the results are presented, where only values with $\gamma_{4}$ remain in the solution space. Following the setting for each parameter, a recipe is formed for the optimized setup of the industrial system.

Comparing the starting conditions of the industrial system with the results achieved for the manual experiments in Lidberg et al. (2018) and to the optimized settings presented in this article, the improvements are presented in Table 3. Optimization enables the simultaneous improvement of several result parameters without manual supervision. This leads to better decision support provided to the decision maker, where she can take decisions considering a range of parameters.

Table 3: Comparison between base values, manual experiments and optimization for ML.

\begin{tabular}{lrrr}
\hline Parameter name & Base & Experiments & Optimization \\
\hline WIP & 10920 & 7914 & 6786 \\
LT & $49 \mathrm{~h}$ & $49 \mathrm{~h}$ & $18 \mathrm{~h}$ \\
Batch sizes & 3100 & 3100 & 1248 \\
\hline
\end{tabular}


Lidberg, Persson, and $\mathrm{Ng}$

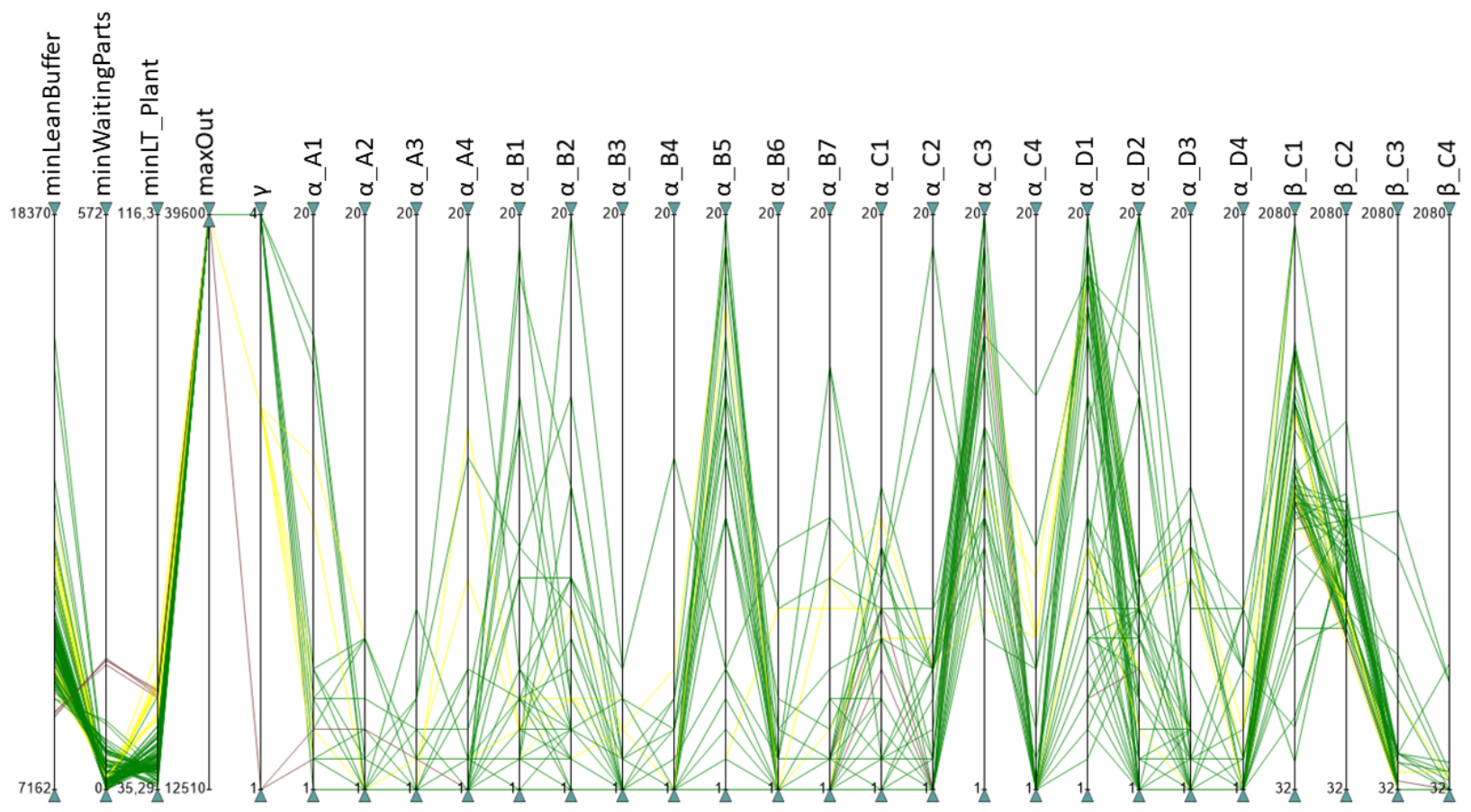

Figure 5: Only selecting solutions on the Pareto front.

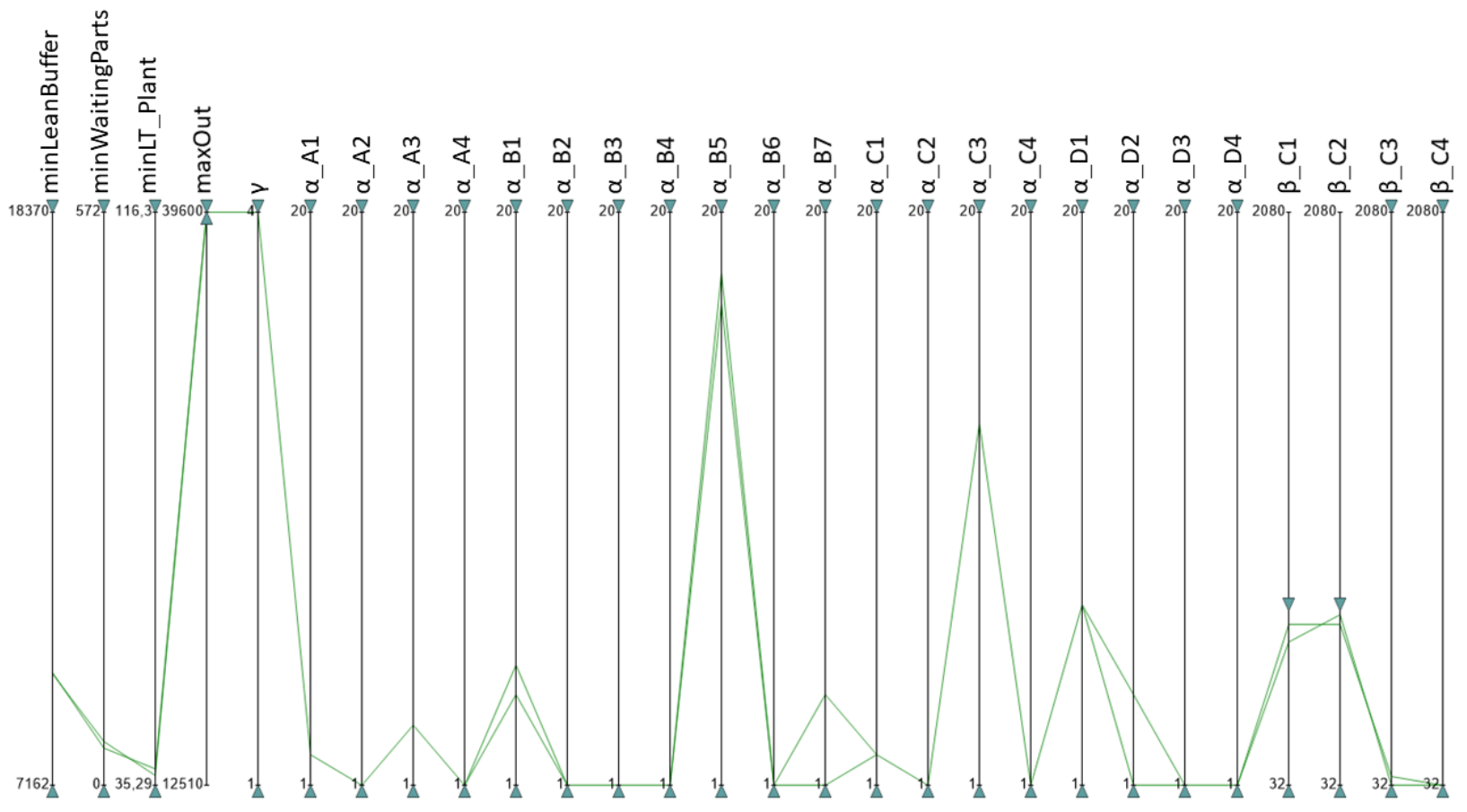

Figure 6: Setting limits on batch sizes. 


\section{CONCLUSIONS AND FUTURE WORK}

This paper shows the potential in utilizing multi-objective optimization together with aggregated DES modeling to enable fast insights into the operation and improvements on an industrial system on the factory level. Compared to previous studies, SMO enables faster delivery of results, with better accuracy, and allows additional input and output parameters to be included. The results from the optimization are also of more value to the decision maker than regular experiments, leading to reductions in the storage levels by $31 \%$, lead time by $67 \%$, and total batch sizes by more than $50 \%$.

Additional work would connect knowledge extraction and data-mining to the optimization to make sure that the conclusions drawn from the optimization data is also of high quality. To improve the industrial system even further, identifying the bottleneck system would be of great interest. Finding the bottleneck and giving suggestions on how to improve it would aid the operations of the industrial system. For the dispatching schedule, testing the advantages of dispatching on weekends is also interesting. In another direction, testing the aggregation technique on an even higher level, i.e. supply chains, would also be of interest.

\section{ACKNOWLEDGMENTS}

The authors express their gratitude to the Swedish Knowledge Foundation and Volvo Car Corporation for party funding the research leading to this paper. Tehseen Aslam and Martin Andersson from the University of Skövde have also contributed assistance with the optimization algorithm and process.

\section{REFERENCES}

Asmundsson, J., R. L. Rardin, and R. Uzsoy. 2006. "Tractable Nonlinear Production Planning Models for Semiconductor Wafer Fabrication Facilities". IEEE Transactions on Semiconductor Manufacturing 19(1):95-111.

Banks, J., B. L. Nelson, J. S. Carson, and D. M. Nicol. 2009. Discrete-Event System Simulation. 5th ed. Essex, UK: Pearson UK.

Chwif, L., M. Barretto, and R. Paul. 2000. "On Simulation Model Complexity". In Proceedings of the 2000 Winter Simulation Conference, edited by J. A. Joines et al., 449-455. Piscataway, New Jersey: IEEE.

Das, I., and J. E. Dennis. 1998. "Normal-Boundary Intersection: A New Method for Generating the Pareto Surface in Nonlinear Multicriteria Optimization Problems". SIAM Journal on Optimization 8(3):631657.

Deb, K. 2001. Multi-Objective Optimization using Evolutionary Algorithms. New York, NY, USA: John Wiley \& Sons.

Deb, K., and H. Jain. 2014. "An Evolutionary Many-Objective Optimization Algorithm using ReferencePoint-based Nondominated Sorting Approach, Part I: Solving Problems with Box Constraints". IEEE Transactions on Evolutionary Computation 18(4):577-601.

Deb, K., A. Pratap, S. Agarwal, and T. Meyarivan. 2002. "A Fast and Elitist Multiobjective Genetic Algorithm: NSGA-II". IEEE Transactions on Evolutionary Computation 6(2):182-197.

Dudas, C., A. H. Ng, L. Pehrsson, and H. Boström. 2014. "Integration of data mining and multi-objective optimisation for decision support in production systems development". International Journal of Computer Integrated Manufacturing 27(9):824-839.

Floreano, D., and C. Mattiussi. 2008. Bio-inspired Artificial Intelligence : Theories, Methods, and Technologies. Cambridge, Massachusetts: MIT Press.

Fowler, J. W., and O. Rose. 2004. "Grand Challenges in Modeling and Simulation of Complex Manufacturing Systems”. Simulation 80(9):469-476.

Hopp, W. J., and M. L. Spearman. 2011. Factory Physics. 3rd ed. Long Grove, IL: Waveland Press, Inc. 


\section{Lidberg, Persson, and $\mathrm{Ng}$}

Jain, H., and K. Deb. 2014. "An Evolutionary Many-Objective Optimization Algorithm using ReferencePoint based Nondominated sorting Approach, Part II: Handling Constraints and Extending to an Adaptive Approach". IEEE Transactions on Evolutionary Computation 18(4):602-622.

Koren, Y. 2010. The Global Manufacturing Revolution: Product-Process-Business Integration and Reconfigurable Systems. Hoboken, New Jersey: John Wiley \& Sons, Inc.

Lefeber, E., and D. Armbruster. 2011. "Aggregate Modeling of Manufacturing Systems". In International Series in Operations Research and Management Science, Volume 151 of International Series in Operations Research \& Management Science, 509-536. Springer, Boston, MA.

Lidberg, S., L. Pehrsson, and M. Frantzén. 2018. “Applying Aggregated Line Modeling Techniques to Optimize Real World Manufacturing Systems". Procedia Manufacturing 25(May):89-96.

Negahban, A., and J. S. Smith. 2014. "Simulation for Manufacturing System Design and Operation: Literature Review and Analysis". Journal of Manufacturing Systems 33(2):241-261.

Ng, A. H., C. Dudas, J. Nießen, and K. Deb. 2011. "Simulation-Based Innovization Using Data Mining for Production Systems Analysis". In Multi-objective Evolutionary Optimisation for Product Design and Manufacturing, edited by L. Wang et al., 401-429. London: Springer London.

Pehrsson, L., M. Frantzen, T. Aslam, and A. H. Ng. 2015. "Aggregated Line Modeling for Simulation and Optimization of Manufacturing Systems". In Proceedings of the 2015 Winter Simulation Conference, edited by L. Yilmaz et al., 3632-3643. Piscataway, New Jersey: IEEE.

Pehrsson, L., S. Lidberg, M. Frantzén, T. Aslam, and A. H. Ng. 2014. "Aggregated Discrete Event Modelling for Simulation and Optimisation of Manufacturing Systems". In 12th International Industrial Simulation Conference 2014, ISC 2014, 83-90: EUROSIS.

Schruben, L. 2010. "Simulation Modeling for Analysis". ACM Transactions on Modeling and Computer Simulation 20(1):1-22.

Zulkepli, J., T. Eldabi, and N. Mustafee. 2012. "Hybrid Simulation for Modelling Large Systems: An example of Integrated Care Model". In Proceedings of the 2012 Winter Simulation Conference, edited by C. Laroque et al., 1-12. Piscataway, New Jersey: IEEE.

\section{AUTHOR BIOGRAPHIES}

SIMON LIDBERG is an industrial Ph.D. student at Volvo Car Corporation and the University of Skövde. Graduating from the University of Skövde in 2011, with a M.Sc. in Industrial Informatics, he started working with the simulation and optimization of production systems using DES. In 2017 he started his industrial $\mathrm{Ph} . \mathrm{D}$. studies focusing on the creation of decision support by optimizing on a factory level using aggregated data from detailed DES models. He can be reached at simon.lidberg@his.se and simon.lidberg@volvocars.com.

LEIF PEHRSSON is the Director of Research and Technology Development, Manufacturing Engineering Power Systems, at Volvo Car Group and an Affiliated Senior Lecturer at the University of Skövde, Sweden. He holds a Ph.D. degree in Manufacturing Systems from De Montfort University in the UK., a M.Sc. degree in Automation Engineering, and a B.Sc. degree in Mechanical Engineering. He has been working in various engineering and managerial positions within automotive industry for more than 20 years. His e-mail address is leif.pehrsson@his.se.

AMOS H.C. NG is a Professor of Production and Automation Engineering at the University of Skövde, Sweden. He holds a Ph.D. degree in Computing Sciences and Engineering. His main research interest lies in applying multi-objective optimization and data mining techniques for production systems design, analysis and improvement. His e-mail address is amos.ng@ his.se. 